NORGES TEKNISK-NATURVITENSKAPELIGE UNIVERSITET

\title{
Goal Oriented Error Estimators for Stokes Equations based on Variationally Consistent Postprocessing
}

by

Hallgeir Melbø and Trond Kvamsdal

\author{
PREPRINT \\ NUMERICS NO. 5/2001
}

\section{NORWEGIAN UNIVERSITY OF SCIENCE AND TECHNOLOGY TRONDHEIM, NORWAY}

This report has URL

http: //www . math.ntnu.no/preprint/numerics/2001/N5-2001.ps

Address: Department of Mathematical Sciences, Norwegian University of Science and

Technology, N-7491 Trondheim, Norway. 



\title{
GOAL ORIENTED ERROR ESTIMATORS FOR STOKES EQUATIONS BASED ON VARIATIONALLY CONSISTENT POSTPROCESSING
}

\author{
HALLGEIR MELB $\varnothing^{1}$ and TROND KVAMSDAL ${ }^{2}$ \\ ${ }^{1}$ Department of Mathematical Sciences, NTNU, N-7491 Trondheim, Norway. \\ email: hallgeir@math.ntnu.no \\ ${ }^{2}$ SINTEF Applied Mathematics, N-7465 Trondheim, Norway. \\ email: Trond.Kvamsdal@math.sintef.no
}

\begin{abstract}
.
In many flow problems, and in particular when fluid-structure interaction is considered, the important unknowns are the forces acting on the structure in certain areas. Hence accurate values for these local quantities is essentially what one wants to get out of the flow computations. By means of Variationally Consistent Postprocessing (VCP), where forces are computed using the weak form of the equations, we recover the requested forces. Goal oriented local error indicators are provided by solving an auxiliary problem. At the end numerical examples are presented that illustrate how this goal oriented strategy gives improved efficiency compared to traditional methods. The fluid flow is assumed to be governed by the Stokes equations.
\end{abstract}

Key words: Stokes Flow, Finite Element Method, Postprocessing, Error Estimation, Adaptivity.

\section{Introduction}

In many applications the primary aim of a finite element analysis is to recover forces or stresses with good accuracy in certain areas. Other quantities may be of little or no interest. In fluid-structure interaction problems, and also in many fluid problems, this is typically the case. It is likely that a uniform refinement of the initial mesh is not an optimal strategy, and also many traditional adaptive techniques are spending grid cells in areas that may not affect the quality of the requested forces. The strategies presented here may be called "goal oriented" in the sense that they are only aiming at getting a good approximation for the forces acting in certain areas which are chosen in advance. Similar strategies have been proposed e.g. in [3], [4], and [6].

In this article we will only consider flows governed by the Stokes equations, which describe very slow viscous fluid flow, often referred to as creeping flow. The concept of Variationally Consistent Postprocessing, proposed in [7], will be 
used to recover the requested forces. This is a way of rewriting a line integral, which expresses the force, as a volume integral by using the weak form of the flow equations. A main point is that we show how this can be done using the Laplacian formulation of the equations.

Finally error estimators are computed after solving an auxiliary (dual) problem. Provided one has robust energy error estimators for both the primal and dual problem, one can obtain an upper bound for the error in the recovered quantity, which converges with optimal rate.

Our theoretical analysis is accompanied by several numerical examples. The examples show that adaptive techniques, and in particular our goal oriented techniques, give very satisfactory results and seem to be superior to standard approaches.

\section{Governing equations}

Let $\Omega \subset \mathbb{R}^{n}, n=2,3$, be an open bounded domain with boundary $\partial \Omega$. Let the boundary be smooth in the sense that it satisfies a Lipschitz condition. The outward pointing normal vector $\mathbf{n}$ will then exist almost everywhere on $\partial \Omega$. Stokes equations for steady state flow in the domain $\Omega$ may be given as

$$
\begin{array}{rlll}
-\nu \Delta \mathbf{u}+\nabla p & =\mathbf{f} & & \text { in } \Omega \\
\nabla \cdot \mathbf{u} & =0 & & \text { in } \Omega .
\end{array}
$$

Here $\mathbf{u}$ is the velocity, $p$ is dynamical pressure, $\mathbf{f}$ is a (scaled) body force, and $\nu$ the (constant) viscosity parameter. The left side of the first equation, often referred to as the momentum equation, may just as well be written as $-\operatorname{div} \sigma$ with $\sigma$ being the stress tensor. This alternative formulation will be discussed in the next section. The equations are equipped with the following boundary conditions,

$$
\begin{aligned}
\mathbf{u} & =\mathbf{g}_{D} & & \text { on } \partial \Omega_{D} \\
\nu \nabla \mathbf{u} \cdot \mathbf{n}-p \mathbf{n} & =\mathbf{g}_{N} & & \text { on } \partial \Omega_{N}
\end{aligned}
$$

where

$$
\nabla \mathbf{u}=\left[\begin{array}{c}
\left(\nabla u_{1}\right)^{T} \\
\vdots \\
\left(\nabla u_{n}\right)^{T}
\end{array}\right]
$$

and

$$
\begin{aligned}
\partial \Omega & =\overline{\partial \Omega}_{D} \cup \overline{\partial \Omega}_{N} \\
\partial \Omega_{D} \cap \partial \Omega_{N} & =\emptyset .
\end{aligned}
$$


Define the following functional spaces,

$$
\begin{aligned}
V^{*} & =\left[H^{1}(\Omega)\right]^{n} \\
U & =\left\{\mathbf{v} \in V^{*}: \mathbf{v}=\mathbf{g}_{D} \text { on } \partial \Omega_{D}\right\} \\
V & =\left\{\mathbf{v} \in V^{*}: \mathbf{v}=0 \text { on } \partial \Omega_{D}\right\} \\
Q & =L^{2}(\Omega)
\end{aligned}
$$

Multiplying the first equation in (2.1) by a test function $\mathbf{v} \in V$ and integrating over the domain gives, with the aid of Green's formula,

$$
a(\mathbf{u}, \mathbf{v})+b(p, \mathbf{v})=(\mathbf{f}, \mathbf{v})+\int_{\partial \Omega}(\nu \nabla \mathbf{u} \cdot \mathbf{n}-p \mathbf{n}) \cdot \mathbf{v} d s
$$

where the different terms are defined as

$$
\begin{aligned}
a(\mathbf{u}, \mathbf{v}) & =\int_{\Omega} \sum_{i=1}^{n} \nu \nabla u_{i} \cdot \nabla v_{i} d x \\
b(p, \mathbf{v}) & =-\int_{\Omega} p \nabla \cdot \mathbf{v} d x \\
(\mathbf{f}, \mathbf{v}) & =\int_{\Omega} \mathbf{f} \cdot \mathbf{v} d x .
\end{aligned}
$$

The line integral in (2.2) is known from the boundary conditions, and the weak form of the Stokes equations becomes: Find $\{\mathbf{u}, p\} \in U \times Q$ such that

$$
\begin{aligned}
a(\mathbf{u}, \mathbf{v})+b(p, \mathbf{v}) & =(\mathbf{f}, \mathbf{v})+\int_{\partial \Omega_{N}} \mathbf{g}_{N} \cdot \mathbf{v} d s & & \forall \mathbf{v} \in V \\
b(q, \mathbf{u}) & =0 & & \forall q \in Q
\end{aligned}
$$

Let $U^{h} \subset U, V^{h} \subset V$, and $Q^{h} \subset Q$ be finite dimensional approximations to the infinite dimensional spaces. The finite element formulation of the Stokes equations becomes: Find $\left\{\mathbf{u}^{h}, p^{h}\right\} \in U^{h} \times Q^{h}$ such that

$$
\begin{aligned}
a\left(\mathbf{u}^{h}, \mathbf{v}^{h}\right)+b\left(p^{h}, \mathbf{v}^{h}\right) & =\left(\mathbf{f}, \mathbf{v}^{h}\right)+\int_{\partial \Omega_{N}} \mathbf{g}_{N} \cdot \mathbf{v}^{h} d s & & \forall v^{h} \in V^{h} \\
b\left(q^{h}, \mathbf{u}^{h}\right) & =0 & & \forall q^{h} \in Q^{h} .
\end{aligned}
$$

If $\partial \Omega_{N}=\emptyset$ the pressure is only unique up to an additional constant, and it may be made unique for instance by requiring the average pressure to vanish.

\section{Discussion of model}

As already mentioned an equivalent model formulation is possible in which $-\operatorname{div} \sigma$ replaces the left hand side of the momentum equation in (2.1). The incompressibility constraint can be used to rewrite any of the formulations as the other. After multiplying by a test function, integrating and applying Green's theorem the stress-formulation gives

$$
\tilde{a}(\mathbf{u}, \mathbf{v})+b(p, \mathbf{v})=(\mathbf{f}, \mathbf{v})+\int_{\partial \Omega}(\hat{\sigma}(\mathbf{u}, p) \cdot \mathbf{n}) \cdot \mathbf{v} d s \quad \forall \mathbf{v} \in V
$$


where

$$
\begin{aligned}
\tilde{a}(\mathbf{u}, \mathbf{v}) & =\int_{\Omega} 2 \nu \nabla^{S} \mathbf{u}: \nabla^{S} \mathbf{v} d x \\
\left(\nabla^{S} \mathbf{v}\right)_{i j} & =\frac{1}{2}\left(\frac{\partial v_{i}}{\partial x_{j}}+\frac{\partial v_{j}}{\partial x_{i}}\right) \\
A: B & =\operatorname{trace}(A \cdot B) \\
\hat{\sigma}(\mathbf{u}, p) & =2 \nu \nabla^{S} \mathbf{u}-I p .
\end{aligned}
$$

The stress tensor is marked with a hat to show that it is divided by the constant density $\rho$, i.e.

$$
\sigma=\rho \hat{\sigma} .
$$

This gives a weak form very similar to the one suggested in the previous section. However, the integrand in the boundary integral is now in general different from its counterpart in (2.2). Whereas the quantity $\sigma \cdot \mathbf{n}$ expresses a traction, which after integration gives a force, this is not necessarily true for the integrand in (2.2). For an exact solution $\left(\mathbf{u}^{*}, p^{*}\right)$ one will therefore in general need different Neumann conditions for the two formulations to make them consistent. In the case of a Laplacian formulation

$$
\mathbf{g}_{N}=\nu \nabla \mathbf{u}^{*} \cdot \mathbf{n}-p^{*} \mathbf{n},
$$

and for the above stress-formulation

$$
\tilde{\mathbf{g}}_{N}=\hat{\sigma}\left(\mathbf{u}^{*}, p^{*}\right) \cdot \mathbf{n}
$$

Both formulations will of course give the same exact solution, but the discrete solutions may differ. The advantage of the stress-formulation is that the Neumann boundary conditions are actual physical surface tractions, while this is in general not the case for the Laplacian formulation. However, the Laplacian formulation has the obvious advantage that it gives a system matrix which decouples into blocks and which has fewer entries than the matrix obtained from the stressformulation.

In later sections we will be computing forces by direct use of the boundary integral appearing in the weak formulation (2.2) when certain test functions are chosen. The computation of such forces is quite straight forward when the stress formulation is used since the boundary integral in (3.1) directly gives a surface force. The question is if this can be accomplished also for the Laplacian formulation, i.e. what kind of condition would imply that the equality

$$
\hat{\sigma}(\mathbf{u}, p) \cdot \mathbf{n}=\nu \nabla \mathbf{u} \cdot \mathbf{n}-p \mathbf{n}
$$

is valid? Whenever this equality holds the integrand in (2.2) expresses a surface traction. The terms involving the pressure are clearly identical so we will consider only the viscous terms and only the $2 \mathrm{D}$ case. The results can be extended 
to $3 \mathrm{D}$, but then the calculations are, although elementary, rather tedious.

Let $\mathbf{u}=\left[\begin{array}{ll}u_{1} & u_{2}\end{array}\right]^{T}$ and $\mathbf{n}=\left[\begin{array}{ll}n_{1} & n_{2}\end{array}\right]^{T}$. Then equation (3.2) requires that

$$
\left[\begin{array}{l}
2 u_{1, x} n_{1}+u_{1, y} n_{2}+u_{2, x} n_{2} \\
u_{1, y} n_{1}+u_{2, x} n_{1}+2 u_{2, y} n_{2}
\end{array}\right]=\left[\begin{array}{l}
u_{1, x} n_{1}+u_{1, y} n_{2} \\
u_{2, x} n_{1}+u_{2, y} n_{2}
\end{array}\right],
$$

in which the subscript ',' indicates differentiation. This implies

$$
\begin{aligned}
& u_{1, x} n_{1}+u_{2, x} n_{2}=0 \\
& u_{1, y} n_{1}+u_{2, y} n_{2}=0 .
\end{aligned}
$$

According to the incompressibility constraint

$$
u_{1, x}+u_{2, y}=0
$$

and thus

$$
\begin{aligned}
& u_{2, x} n_{2}-u_{2, y} n_{1}=0 \\
& u_{1, x} n_{2}-u_{1, y} n_{1}=0
\end{aligned}
$$

which is equivalent to

$$
\nabla \mathbf{u} \cdot \mathbf{t}=0
$$

where $\mathbf{t}=\left[n_{2},-n_{1}\right]^{T}$ is the tangent vector. Therefore, if the gradient in the tangential direction vanishes the line integral in the Laplacian formulation equals its counterpart in the stress formulation, in which case the line integral expresses a force.

The requirement (3.3) is satisfied for instance on no-slip walls, and hence the integrand in the boundary integral in (2.2) expresses a traction on walls. Let $\mathbf{d}$ be a vector of unit length. Then the (scaled) force $\hat{F}_{\mathbf{d}}$ in direction $\mathbf{d}$ acting on a no-slip wall with boundary $\Gamma$ can be expressed as

$$
\hat{F}_{\mathbf{d}}=-\int_{\Gamma}(\hat{\sigma}(\mathbf{u}, p) \cdot \mathbf{n}) \cdot \mathbf{d} d s=-\int_{\Gamma}(\nu \nabla \mathbf{u} \cdot \mathbf{n}-p \mathbf{n}) \cdot \mathbf{d} d s
$$

where we recall that $p$ is the dynamical pressure, i.e. it is the actual pressure divided by $\rho$.

\section{Variationally Consistent Postprocessing}

The term Variationally Consistent Postprocessing (VCP) was introduced in [7] and it describes the concept of applying the weak formulation of the problem to postprocess the computed data. This is done in order to obtain high accuracy in the computed quantities, see proof in [6]. 
We will here consider forces acting on no-slip walls, and according to the results in the previous section the scaled force $\hat{F}$ in direction $\mathbf{d}$ acting on $\partial \Omega_{R} \subset \Omega_{D}$ may be written

$$
-\hat{F}(\mathbf{u}, p)=\int_{\partial \Omega_{R}} \nu(\nabla \mathbf{u} \cdot \mathbf{n}) \cdot \mathbf{d} d s
$$

where $\mathbf{d}$ has unit length.

As the differential equation (2.1) is assumed to hold, the weak formulation (2.2) will hold also for test functions that do not satisfy the Dirichlet boundary conditions for the space $V$. In a more general setting we can even open for test functions from what is usually referred to as the broken space. This space contains functions that are in $V^{*}$ inside each element but only in $L^{2}$ across element boundaries. The bilinear forms then need to be substituted by mesh dependent forms in the following manner,

$$
c^{h}(\cdot, \cdot):=\sum_{K=1}^{N_{\text {elm }}} c_{K}(\cdot, \cdot)=\left.\sum_{K=1}^{N_{\text {elm }}} c(\cdot, \cdot)\right|_{\Omega_{K}},
$$

where the general bilinear form $c(\cdot, \cdot)$ can be either $a(\cdot, \cdot)$ or $b(\cdot, \cdot)$. These mesh dependent forms coincide with the original bilinear forms when they operate on functions in $U$ or $V$. (For the space $Q$ the original space and the broken space are identical.) We will not be considering functions from the broken space, but for generality we will follow [7] and use the mesh dependent forms.

Test functions that do not respect the boundary conditions in $V$ can be exploited to rewrite the line integral (4.1) as a volume integral. Assume now that the test function is chosen as a function from the finite element space except that it equals the directional vector $\mathbf{d}$ on the part of the boundary, $\partial \Omega_{R}$, where the force is to be recovered. Then it is also necessarily nonzero on a part of $\partial \Omega$ that is connected to and in the vicinity of $\partial \Omega_{R}$. The chosen test function will be called an extraction function and it is in general a vector $\mathbf{w}^{T}=\left[\mathbf{w}_{u}^{T}, w_{p}\right]$. Here $\mathbf{w}_{u}$ corresponds to velocity and $w_{p}$ corresponds to pressure. The component $w_{p}$ is in fact redundant and it may be set to zero. As will be seen later, $w_{p}$ is only kept for clarity and it will be assumed to be in the space $Q$. This makes $w_{p} \equiv 0$ a perfectly valid choice. Using this extraction function as a test function shows that

$$
\begin{aligned}
a^{h}\left(\mathbf{u}, \mathbf{w}_{u}\right)+b^{h}\left(p, \mathbf{w}_{u}\right)-\left(\mathbf{f}, \mathbf{w}_{u}\right) & =\int_{\partial \Omega}(\nu \nabla \mathbf{u} \cdot \mathbf{n}-p \mathbf{n}) \cdot \mathbf{w}_{u} d s \\
& =\int_{\partial \Omega_{R}} \nu(\nabla \mathbf{u} \cdot \mathbf{n}) \cdot \mathbf{d} d s+\int_{\partial \Omega \backslash \partial \Omega_{R}}(\nu \nabla \mathbf{u} \cdot \mathbf{n}-p \mathbf{n}) \cdot \mathbf{w}_{u} d s .
\end{aligned}
$$

This suggests a way of expressing the line integral as a volume integral,

$$
-\hat{F}(\mathbf{u}, p)=a^{h}\left(\mathbf{u}, \mathbf{w}_{u}\right)+b^{h}\left(p, \mathbf{w}_{u}\right)-\left(\mathbf{f}, \mathbf{w}_{u}\right)-\int_{\partial \Omega \backslash \partial \Omega_{R}}(\nu \nabla \mathbf{u} \cdot \mathbf{n}-p \mathbf{n}) \cdot \mathbf{w}_{u} d s .
$$


When evaluating the force numerically the volume integral usually gives a more robust evaluation, and as will be seen later, this also gives optimal rate of convergence for the computed quantity in the sense that it converges with the same rate as the square of the energy.

However, if $\mathbf{w}_{u}$ is nonzero on $\partial \Omega \backslash \partial \Omega_{R}$ one still needs to compute line integrals to evaluate $\hat{F}$, but this can be limited by letting w satisfy the following conditions,

$$
\begin{aligned}
\operatorname{supp}\left(\mathbf{w}_{u}\right) & =\omega \subset \Omega \\
\partial \Omega_{R} & \subset \partial \omega \\
\partial \Omega_{R} & \subset \partial \Omega_{D} \\
\mathbf{w}_{u} & \in \tilde{V}^{h} \subset \tilde{V} \\
\tilde{V} & =\left\{\mathbf{v} \in V^{*}: \mathbf{v}=0 \text { on } \partial \Omega \backslash \partial \omega, \mathbf{v}=\mathbf{d} \text { on } \partial \Omega_{R}\right\} \\
w_{p} & \in Q^{h}
\end{aligned}
$$

where $\tilde{V}^{h}$ and $V^{h}$ are identical except for the boundary conditions. Note that the analysis will be restricted to the recovery of forces on $\partial \Omega_{D}$. We define $\Gamma_{0}$ as the part of $\partial \Omega$ where the line integrals in (4.2) still need to be evaluated, i.e.

$$
\Gamma_{0}:\left\{\begin{array}{l}
\Gamma_{0} \subset \partial \omega \backslash \partial \Omega_{R} \\
\left.\mathbf{w}_{u}\right|_{\Gamma_{0}} \neq 0
\end{array}\right.
$$

The recovery operator $R_{\mathbf{w}}: V^{*} \times Q \rightarrow \mathbb{R}$ is now defined by

$$
R_{\mathbf{w}}(\mathbf{v}, q)=a^{h}\left(\mathbf{v}, \mathbf{w}_{u}\right)+b^{h}\left(q, \mathbf{w}_{u}\right)-\left(\mathbf{f}, \mathbf{w}_{u}\right)-\int_{\Gamma_{0}}(\nu \nabla \mathbf{v} \cdot \mathbf{n}-q \mathbf{n}) \cdot \mathbf{w}_{u} d s .
$$

With this definition of $R_{\mathbf{w}}$,

$$
\begin{aligned}
R_{\mathbf{w}}(\mathbf{u}, p) & =-\hat{F}(\mathbf{u}, p) \\
& =a^{h}\left(\mathbf{u}, \mathbf{w}_{u}\right)+b^{h}\left(p, \mathbf{w}_{u}\right)-\left(\mathbf{f}, \mathbf{w}_{u}\right)-\int_{\Gamma_{0}}(\nu \nabla \mathbf{u} \cdot \mathbf{n}-p \mathbf{n}) \cdot \mathbf{w}_{u} d s .
\end{aligned}
$$

Since the actual solution is unknown, $R_{\mathbf{w}}(\mathbf{u}, p)$ is approximated by $R_{\mathbf{w}}\left(\mathbf{u}^{h}, p^{h}\right)$ when the force is computed. A simple $2 \mathrm{D}$ example of this concept is shown in Figure 4.1, where the force to be recovered is a force in the $\mathrm{x}$-direction acting on a part of the boundary that is parallel to the $\mathrm{x}$-axis. The second component of $\mathbf{w}_{u}$ is then zero, $w_{p}$ may be chosen to be zero, and the first component is shown in the figure. Here we can see that there are still two parts of $\partial \omega$, that are not part of $\partial \Omega_{R}$, where $\mathbf{w}_{u}$ is nonzero, i.e. $\Gamma_{0} \neq \emptyset$. These parts will contribute to the last term in (4.5) and it seems like nothing is gained since line integrals are still present. However, in many cases the unknown forces are acting on fully immersed bodies, i.e. $\Gamma_{0}=\emptyset$ such that these extra integrals vanish. For cases like in Figure 4.1, the domain $\omega$ may be chosen very narrow at the ends making $\left|\Gamma_{0}\right|$ small. A small relative error in direct computations of the two line integrals 


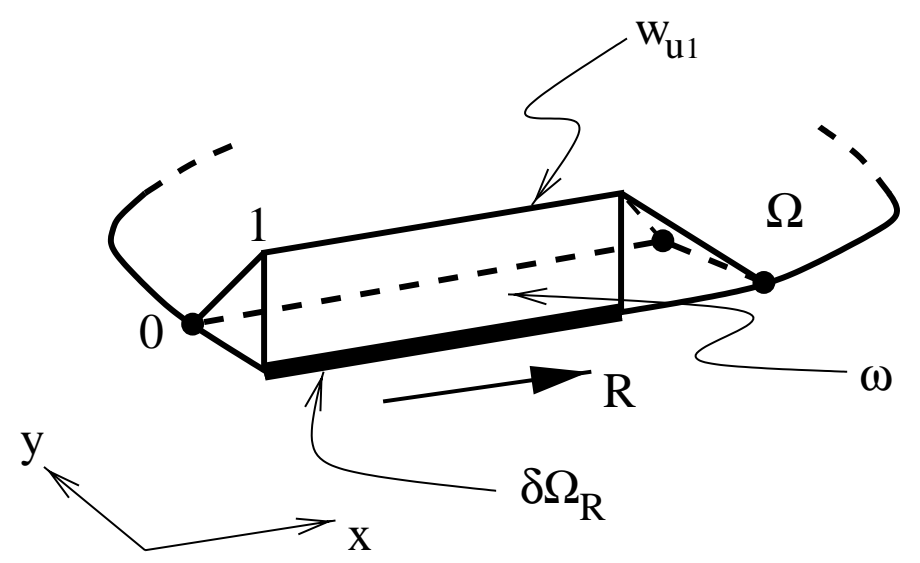

Figure 4.1: An extraction function for extracting a force in the x-direction on a part of $\partial \Omega$ that is parallel to the $\mathrm{x}$-axis. Only the first component of $\mathbf{w}$ is nonzero.

will then in most cases make a negligible contribution to $R_{\mathbf{w}}$.

Exactly the same technique can be applied when using the stress-formulation, and with that formulation this technique has also been described for the NavierStokes equations [8]. A more detailed description of VCP is found in [7].

\section{Goal oriented adaptive strategies}

\subsection{Goal orientation}

As already mentioned, the interesting output of many flow simulations are certain local surface forces. We therefore define our goal as to "compute a local surface force with a prescribed level of accuracy using as few finite elements as possible". That means we are not concerned about getting the overall error small or getting a correct flow field throughout the domain $\Omega$. In this section we will develop adaptive strategies that are aimed directly towards this goal.

\subsection{Error representation and dual problem}

The fact that one has to use the finite element solution instead of the true solution when recovering the force introduces the following error,

$$
\begin{aligned}
E_{\mathbf{w}}\left(\mathbf{u}, \mathbf{u}^{h}, p, p^{h}\right) & =R_{\mathbf{w}}(\mathbf{u}, p)-R_{\mathbf{w}}\left(\mathbf{u}^{h}, p^{h}\right) \\
& =a^{h}\left(\mathbf{e}_{u}, \mathbf{w}_{u}\right)+b^{h}\left(e_{p}, \mathbf{w}_{u}\right)-\int_{\Gamma_{0}}\left\{\nu \nabla \mathbf{e}_{u} \cdot \mathbf{n}-e_{p} \mathbf{n}\right\} \cdot \mathbf{w}_{u} d s,
\end{aligned}
$$

where

$$
\begin{aligned}
& \mathbf{e}_{u}=\mathbf{u}-\mathbf{u}^{h} \\
& e_{p}=p-p^{h} .
\end{aligned}
$$


Hence, by defining the error functionals $E_{R}: V^{*} \times Q \times V^{*} \times Q \rightarrow \mathbb{R}$ and $E_{\Gamma_{0}}: V^{*} \times Q \times V^{*} \rightarrow \mathbb{R}$ as

$$
\begin{aligned}
E_{R}\left(\mathbf{v}, q, \mathbf{w}_{v}, w_{q}\right) & =a^{h}\left(\mathbf{v}, \mathbf{w}_{v}\right)+b^{h}\left(q, \mathbf{w}_{v}\right)+b^{h}\left(w_{q}, \mathbf{v}\right) \\
E_{\Gamma_{0}}\left(\mathbf{v}, q, \mathbf{w}_{v}\right) & =-\int_{\Gamma_{0}}\{\nu \nabla \mathbf{v} \cdot \mathbf{n}-q \mathbf{n}\} \cdot \mathbf{w}_{v} d s
\end{aligned}
$$

the error may be written as

$$
E_{\mathbf{w}}\left(\mathbf{u}, \mathbf{u}^{h}, p, p^{h}\right)=E_{R}\left(\mathbf{e}_{u}, e_{p}, \mathbf{w}_{u}, w_{p}\right)+E_{\Gamma_{0}}\left(\mathbf{e}_{u}, e_{p}, \mathbf{w}_{u}\right) .
$$

Note that here an extra $b(\cdot, \cdot)$ is added, but since $w_{p} \in Q^{h}$ this gives only a zero contribution because of the incompressibility constraint.

Let $\left\{\mathbf{z}_{u}, z_{p}\right\} \in V \times Q$ satisfy the following dual problem,

$$
\begin{aligned}
a\left(\mathbf{v}, \mathbf{z}_{u}\right)+b\left(z_{p}, \mathbf{v}\right) & =a^{h}\left(\mathbf{v}, \mathbf{w}_{u}\right)+b^{h}\left(w_{p}, \mathbf{v}\right) & & \forall \mathbf{v} \in V \\
b\left(q, \mathbf{z}_{u}\right) & =b^{h}\left(q, \mathbf{w}_{u}\right) & & \forall q \in Q .
\end{aligned}
$$

Then

$$
\begin{aligned}
E_{R}\left(\mathbf{v}, q, \mathbf{w}_{u}, w_{p}\right) & =a^{h}\left(\mathbf{v}, \mathbf{w}_{u}\right)+b^{h}\left(q, \mathbf{w}_{u}\right)+b^{h}\left(w_{p}, \mathbf{v}\right) \\
& =a\left(\mathbf{v}, \mathbf{z}_{u}\right)+b\left(q, \mathbf{z}_{u}\right)+b\left(z_{p}, \mathbf{v}\right) \\
& =E_{R}\left(\mathbf{v}, q, \mathbf{z}_{u}, z_{p}\right)
\end{aligned}
$$

and therefore

$$
E_{R}\left(\mathbf{e}_{u}, e_{p}, \mathbf{w}_{u}, w_{p}\right)=E_{R}\left(\mathbf{e}_{u}, e_{p}, \mathbf{z}_{u}, z_{p}\right)
$$

The Galerkin orthogonality states that

$$
E_{R}\left(\mathbf{e}_{u}, e_{p}, \mathbf{z}_{u}^{h}, w_{p}^{h}\right)=0
$$

for any $\left\{\mathbf{z}_{u}^{h}, w_{p}^{h}\right\} \in V^{h} \times Q^{h}$. Denote by $\mathcal{P}$ the finite element partition of $\Omega$ and for simplicity let

$$
E_{R} \equiv E_{R}\left(\mathbf{e}_{u}, e_{p}, \mathbf{w}_{u}, w_{p}\right) .
$$

Utilising the Galerkin orthogonality and the Cauchy-Schwarz inequality we then 
obtain

$$
\begin{aligned}
& \left|E_{R}\right|=\left|\sum_{K \in \mathcal{P}} a_{K}\left(\mathbf{e}_{u}, \mathbf{z}_{u}-\mathbf{z}_{u}^{h}\right)+b_{K}\left(e_{p}, \mathbf{z}_{u}-\mathbf{z}_{u}^{h}\right)+b_{K}\left(z_{p}-z_{p}^{h}, \mathbf{e}_{u}\right)\right| \\
& \leq \sum_{K \in \mathcal{P}} \nu\left|\mathbf{e}_{u}\right|_{H^{1}(K)}\left|\mathbf{z}_{u}-\mathbf{z}_{u}^{h}\right|_{H^{1}(K)}+\left\|e_{p}\right\| K\left\|\nabla \cdot\left(\mathbf{z}_{u}-\mathbf{z}_{u}^{h}\right)\right\|_{K} \\
& +\left\|z_{p}-z_{p}^{h}\right\|_{K}\left\|\nabla \cdot\left(\mathbf{e}_{u}\right)\right\|_{K} \\
& \leq C \sum_{K \in \mathcal{P}} \sqrt{\left|\mathbf{e}_{u}\right|_{H^{1}(K)}^{2}+\left\|e_{p}\right\|_{K}^{2}+\left\|\nabla \cdot \mathbf{u}^{h}\right\|_{K}^{2}} \\
& \cdot \sqrt{\left|\mathbf{z}_{u}-\mathbf{z}_{u}^{h}\right|_{H^{1}(K)}^{2}+\left.\left\|z_{p}-z_{p}^{h}||_{K}^{2}+\right\| \nabla \cdot\left(\mathbf{z}_{u}-\mathbf{z}_{u}^{h}\right)\right|_{K} ^{2}} \\
& \leq C \sum_{K \in \mathcal{P}} \sqrt{\left\|\mathbf{e}_{u}\right\|_{H^{1}(K)}^{2}+\left\|e_{p}\right\|_{K}^{2}+\left\|\nabla \cdot \mathbf{u}^{h}\right\|_{K}^{2}} \\
& \cdot \sqrt{\left\|\mathbf{z}_{u}-\mathbf{z}_{u}^{h}\right\|_{H^{1}(K)}^{2}+\left\|z_{p}-z_{p}^{h}\right\|_{K}^{2}+\|\left.\nabla \cdot\left(\mathbf{w}_{u}-\mathbf{z}_{u}^{h}\right)\right|_{K} ^{2}}
\end{aligned}
$$

The last inequality deserves a comment. Choosing $q=\nabla \cdot\left(\mathbf{z}_{u}-\mathbf{w}_{u}\right)$ in (5.4) leads to

$$
\left\|\nabla \cdot\left(\mathbf{z}_{u}-\mathbf{w}_{u}\right)\right\|_{K}=0
$$

and hence, by the triangle inequality,

$\left.\left\|\left.\nabla \cdot\left(\mathbf{z}_{u}-\mathbf{z}_{u}^{h}\right)\right|_{K} \leq\right\| \nabla \cdot\left(\mathbf{z}_{u}-\mathbf{w}_{u}\right)\left\|_{K}+\right\| \nabla \cdot\left(\mathbf{w}_{u}-\mathbf{z}_{u}^{h}\right)\right|_{K}=\left\|\nabla \cdot\left(\mathbf{w}_{u}-\mathbf{z}_{u}^{h}\right)\right\|_{K}$.

Similar use of the triangle inequality shows that this inequality holds as an equality. As will be seen later, it is important that $\mathbf{z}_{u}$ can be substituted by $\mathbf{w}_{u}$ here. Let $\left\{\tilde{\mathbf{z}}_{u}, \tilde{z}_{p}\right\} \in V^{*} \times Q$ satisfy the following alternative formulation of the dual problem,

$$
\left.\begin{array}{rlrl}
a\left(\mathbf{v}, \tilde{\mathbf{z}}_{u}\right)+b\left(\tilde{z}_{p}, \mathbf{v}\right) & =0 & & \forall \mathbf{v} \in V \\
b\left(q, \tilde{\mathbf{z}}_{u}\right) & =0 & & \forall q, \in Q \\
\tilde{\mathbf{z}}_{u} & =\mathbf{w}_{u} & & \text { on } \partial \Omega_{D},
\end{array}\right\}
$$

where

$$
\begin{aligned}
\tilde{\mathbf{z}}_{u} & =\mathbf{w}_{u}-\mathbf{z}_{u} \\
\tilde{z}_{p} & =w_{p}-z_{p} .
\end{aligned}
$$

Let $\left\{\tilde{\mathbf{z}}_{u}^{h}, \tilde{z}_{p}^{h}\right\} \in \tilde{V}^{h} \times Q^{h}$ be the finite element solution to (5.7), where $\tilde{V}^{h}$ equals $V^{h}$ except for the obvious difference in boundary conditions on $\partial \Omega_{D}$. Then error estimators $\hat{\eta}_{K}, \hat{\eta}$ and $\tilde{\eta}_{K}, \tilde{\eta}$ for the primal and dual problem, respectively, will be referred to as robust if there exists a constant $C$, depending only on the geometry, such that

$$
\begin{aligned}
\left\|\mathbf{u}-\mathbf{u}^{h}\right\|_{H^{1}(\Omega)}^{2}+\left\|p-p^{h}\right\|_{\Omega}^{2}+\left\|\nabla \cdot \mathbf{u}^{h}\right\|_{\Omega}^{2} & \leq C \hat{\eta}^{2} \\
\left\|\tilde{\mathbf{z}}_{u}-\tilde{\mathbf{z}}_{u}^{h}\right\|_{H^{1}(\Omega)}^{2}+\left\|\tilde{z}_{p}-\tilde{z}_{p}^{h}\right\|_{\Omega}^{2}+\left\|\nabla \cdot \tilde{\mathbf{z}}_{u}^{h}\right\|_{\Omega}^{2} & \leq C \tilde{\eta}^{2}
\end{aligned}
$$


and

$$
\begin{aligned}
& \hat{\eta}^{2} \leq C\left\{\left\|\mathbf{u}-\mathbf{u}^{h}\right\|_{H^{1}(\Omega)}^{2}+\left\|p-p^{h}\right\|_{\Omega}^{2}+\left\|\nabla \cdot \mathbf{u}^{h}\right\|_{\Omega}^{2}\right\} \\
& \tilde{\eta}^{2} \leq C\left\{\left\|\tilde{\mathbf{z}}_{u}-\tilde{\mathbf{z}}_{u}^{h}\right\|_{H^{1}(\Omega)}^{2}+\left\|\tilde{z}_{p}-\tilde{z}_{p}^{h}\right\|_{\Omega}^{2}+\left\|\nabla \cdot \tilde{\mathbf{z}}_{u}^{h}\right\|_{\Omega}^{2}\right\},
\end{aligned}
$$

where

$$
\begin{aligned}
\hat{\eta}^{2} & =\sum_{K \in \mathcal{P}} \hat{\eta}_{K}^{2} \\
\tilde{\eta}^{2} & =\sum_{K \in \mathcal{P}} \tilde{\eta}_{K}^{2} .
\end{aligned}
$$

The error associated with the line integrals satisfies

$$
\begin{aligned}
\left|E_{\Gamma_{0}}\left(\mathbf{e}_{u}, e_{p}, \mathbf{w}_{u}\right)\right| & =\left|\int_{\Gamma_{0}}\left\{\nu \nabla \mathbf{e}_{u} \cdot \mathbf{n}-e_{p} \mathbf{n}\right\} \cdot \mathbf{w}_{u} d s\right| \\
& \leq\left\{\nu\left\|\nabla \mathbf{e}_{u} \cdot \mathbf{n}\right\|_{\Gamma_{0}}+\left\|e_{p} \mathbf{n}\right\| \Gamma_{\Gamma_{0}}\right\}\left\|\mathbf{w}_{u}\right\|_{\Gamma_{0}} \\
& \leq C\left\{\left\|\nabla \mathbf{e}_{u} \cdot \mathbf{n}\right\|_{\Gamma_{0}}+\left\|e_{p}\right\|_{\Gamma_{0}}\right\}
\end{aligned}
$$

since all components of $\mathbf{w}_{u}$ can be chosen less than some constant. Note that if $N$ is the number of degrees of freedom in the finite element formulation and $n$ is the number of dimensions then, on a quasi uniform mesh, we have

$$
\mathcal{O}\left(h^{k}\right)=\mathcal{O}\left(N^{-k / n}\right)
$$

where $h$ is the maximum length scale in the mesh.

Lemma 5.1 Let $\{\mathbf{u}, p\}$ satisfy (2.3), let $\left\{\tilde{\mathbf{z}}_{u}, \tilde{z}_{p}\right\}$ satisfy (5.7), and let $\mathbf{u}^{h}, p^{h}, \tilde{\mathbf{z}}_{u}^{h}, \tilde{z}_{p}^{h}$ be the corresponding finite element approximations. Assume that the primal and dual problem have convergence rate $k$, i.e. there exists a constant $C$, depending on the geometry, such that

$$
\begin{aligned}
\left\|\mathbf{u}-\mathbf{u}^{h}\right\|_{H^{1}(\Omega)}^{2}+\left\|p-p^{h}\right\|_{\Omega}^{2}+\left\|\nabla \cdot \mathbf{u}^{h}\right\|_{\Omega}^{2} & \leq C N^{-2 k / n} \\
\left\|\tilde{\mathbf{z}}_{u}-\tilde{\mathbf{z}}_{u}^{h}\right\|_{H^{1}(\Omega)}^{2}+\left\|\tilde{z}_{p}-\tilde{z}_{p}^{h}\right\|_{\Omega}^{2}+\left\|\nabla \cdot \tilde{\mathbf{z}}_{u}^{h}\right\|_{\Omega}^{2} & \leq C N^{-2 k / n}
\end{aligned}
$$

where $N$ is the number of degrees of freedom in the finite element formulation and $n$ is the number of dimensions. Let $\hat{\eta}_{K}, \hat{\eta}$ and $\tilde{\eta}_{K}, \tilde{\eta}$ be robust estimators, in the sense of (5.9) and (5.10), for the primal and dual problem respectively, and define

$$
\eta_{K}=\max \left\{\hat{\eta}_{K}, \tilde{\eta}_{K}\right\} \quad \eta^{2}=\sum_{K \in \mathcal{P}} \eta_{K}^{2}
$$

Then there exists a constant $C$ such that

$$
\left|E_{R}\right| \leq C \eta^{2} \leq C N^{-2 k / n}
$$


where $E_{R} \equiv E_{R}\left(\mathbf{e}_{u}, e_{p}, \mathbf{w}_{u}, w_{p}\right)$ is defined in (5.1), $\mathbf{e}_{u}$ and $e_{p}$ are defined in (5.2), and $\left[\mathbf{w}_{u}, w_{p}\right]$ is the extraction function satisfying (4.3). Moreover, there exists a constant $C$ such that

$$
\left|E_{\mathbf{w}}\left(\mathbf{u}, \mathbf{u}^{h}, p, p^{h}\right)\right| \leq\left|E_{R}\right|+C\left\{\left\|\nabla \mathbf{e}_{u} \cdot \mathbf{n}||_{\Gamma_{0}}+\right\| e_{p} \|_{\Gamma_{0}}\right\},
$$

where $\Gamma_{0}$ is defined in (4.4).

PROOF. Similarly to (5.8), let $\mathbf{z}_{u}^{h}$ and $z_{p}^{h}$ be defined through the following relations,

$$
\begin{aligned}
\tilde{\mathbf{z}}_{u}^{h} & =\mathbf{w}_{u}-\mathbf{z}_{u}^{h} \\
\tilde{z}_{p}^{h} & =w_{p}-z_{p}^{h},
\end{aligned}
$$

where $\left\{\tilde{\mathbf{z}}_{u}^{h}, \tilde{z}_{p}^{h}\right\}$ is the finite element solution to (5.7). Then

$$
\begin{aligned}
\left\|\mathbf{z}_{u}-\mathbf{z}_{u}^{h}\right\|_{H^{1}(\Omega)}^{2}+\left\|z_{p}-z_{p}^{h}\right\|_{\Omega}^{2}+\left\|\nabla \cdot\left(\mathbf{w}_{u}-\mathbf{z}_{u}^{h}\right)\right\|_{\Omega}^{2} \\
\quad=\left\|\tilde{\mathbf{z}}_{u}-\tilde{\mathbf{z}}_{u}^{h}\right\|_{H^{1}(\Omega)}^{2}+\left\|\tilde{z}_{p}-\tilde{z}_{p}^{h}\right\|_{\Omega}^{2}+\left\|\nabla \cdot \tilde{\mathbf{z}}_{u}^{h}\right\|_{\Omega}^{2} .
\end{aligned}
$$

For simplicity define

$$
\begin{aligned}
\hat{\xi}_{K}^{2} & =\left\|\mathbf{u}-\mathbf{u}^{h}\right\|_{H^{1}(K)}^{2}+\left\|p-p^{h}\right\|_{K}^{2}+\left\|\nabla \cdot \mathbf{u}^{h}\right\|_{K}^{2} \\
\tilde{\xi}_{K}^{2} & =\left\|\tilde{\mathbf{z}}_{u}-\tilde{\mathbf{z}}_{u}^{h}\right\|_{H^{1}(K)}^{2}+\left\|\tilde{z}_{p}-\tilde{z}_{p}^{h}\right\|_{K}^{2}+\left\|\nabla \cdot \tilde{\mathbf{z}}_{u}^{h}\right\|_{K}^{2} \\
\xi_{K} & =\max \left\{\hat{\xi}_{K}, \tilde{\xi}_{K}\right\},
\end{aligned}
$$

and let

$$
\hat{\xi}^{2}=\sum_{K \in \mathcal{P}} \hat{\xi}_{K}^{2} \quad \tilde{\xi}^{2}=\sum_{K \in \mathcal{P}} \tilde{\xi}_{K}^{2} \quad \xi^{2}=\sum_{K \in \mathcal{P}} \xi_{K}^{2} .
$$

Then, from (5.6) and (5.14) we obtain

$$
\left|E_{R}\right| \leq \sum_{K \in \mathcal{P}} \hat{\xi}_{K} \tilde{\xi}_{K} \leq \xi^{2} .
$$

Since the estimators are assumed to be robust we have

$$
\xi^{2} \leq C\left\{\hat{\eta}^{2}+\tilde{\eta}^{2}\right\} \leq C \eta^{2}
$$

and

$$
\eta^{2} \leq \hat{\eta}^{2}+\tilde{\eta}^{2} \leq C\left\{\hat{\xi}^{2}+\tilde{\xi}^{2}\right\} .
$$

Utilising the fact that the primal and the dual problem both have convergence rate $k$ completes the proof for the first statement. The second statement follows directly from (5.3) and (5.11). 


\section{Remarks:}

1. The first inequality in (5.13) holds independently of equation (5.10) and the convergence rate assumption.

2. Estimators satisfying (5.9) and (5.10) can be found in the literature. In the examples the following explicit estimator will be applied,

$$
\begin{aligned}
\hat{\eta}_{K}^{2}=h_{K}^{2} \|-\nu \Delta \mathbf{u}^{h} & +\nabla p^{h}-\mathbf{f} \|_{K}^{2} \\
& +\frac{1}{2} \sum_{\Gamma \in \partial K} h_{\Gamma}\left\|\left[\nu \nabla \mathbf{u}^{h} \cdot \mathbf{n}-p^{h} \mathbf{n}\right]\right\|_{\Gamma}^{2}+\left\|\nabla \cdot \mathbf{u}^{h}\right\|_{K}^{2},
\end{aligned}
$$

where $h_{K}$ is the element length scale and $h_{\Gamma}$ is the edge length scale. The same estimator can now also be used for the dual problem, in which $\mathbf{f}=0$ and $\mathbf{u}^{h}$ and $p^{h}$ are replaced by $\tilde{\mathbf{z}}_{u}^{h}$ and $\tilde{z}_{p}^{h}$. This was the reason for changing from $\left\{\mathbf{z}_{u}, z_{p}\right\}$ to $\left\{\tilde{\mathbf{z}}_{u}, \tilde{z}_{p}\right\}$. These estimators are robust, see e.g. [10].

3 . If the error estimators $\hat{\eta}_{K}$ and $\tilde{\eta}_{K}$ are good estimators for the quantities $\hat{\xi}_{K}$ and $\tilde{\xi}_{K}$ defined in the proof of Lemma 5.1, the following should be a suitable estimator for the error in the recovered force,

$$
\left(\eta_{K}^{*}\right)^{2}=\hat{\eta}_{K} \tilde{\eta}_{K} \quad\left(\eta^{*}\right)^{2}=\sum_{K \in \mathcal{P}}\left(\eta_{K}^{*}\right)^{2} .
$$

Unless $\hat{\eta}_{K}$ and $\tilde{\eta}_{K}$ are upper bounds for $\hat{\xi}_{K}$ and $\tilde{\xi}_{K}$, this estimator will not give an upper bound for the error $E_{R}$. Estimators that guarantee this property are yet to be developed. However, since $\hat{\eta}_{K}$ and $\tilde{\eta}_{K}$ often approximate $\hat{\xi}_{K}$ and $\tilde{\xi}_{K}$ well, it is likely that $\eta^{*}$ will work well both as an error estimator and as a refinement criterion.

Note that the dual problem (5.7) is simply the same Stokes problem as (2.3) but with a different right hand side. Existence and uniqueness for the dual problem therefore follows directly from the same properties of the original problem, see e.g. [5]. Since the system matrix for the dual problem is identical to the system matrix for the primal problem, the dual can be solved almost for free if the matrix is factorised or a good preconditioner is made when solving the original problem.

\section{Numerical examples}

In all the examples $\nu=1$ and all examples are in 2D. If the recovered forces correspond to a closed line integral there will be no line integrals present in (4.5), i.e. $\Gamma_{0}=\emptyset$. When this is not the case the values for the line integrals are replaced by "exact" values before the results are plotted. The "exact" values are taken to be the values computed on the finest grid since $\mathbf{w}$ is kept fixed throughout the adaptive procedures.

In many of the convergence plots in this section there are dashed lines indicating rates of convergence. They are typically labelled with $\mathcal{O}\left(h^{k}\right)$. What these 
lines actually show is $\mathcal{O}\left(\frac{1}{N^{k / 2}}\right)$ where $N$ is the number of degrees of freedom. The measure $h$ does not make much sense for graded grids, but the notation is applied because this is a standard way of showing convergence plots.

Two different finite element formulations will be applied. One uses a mixed triangular element where the velocities are interpolated by continuous second order polynomials and the pressure is interpolated by continuous first order polynomials. This is usually referred to as a Taylor-Hood element. It is a stable element, and the energy error estimator $\hat{\eta}_{K}$ defined in (5.15) can be applied.

The other formulation is a first order triangular element, where the velocity and the pressure are interpolated by continuous first order polynomials. This formulation requires stabilisation, and the following formulation is therefore applied,

$$
\begin{aligned}
a\left(\mathbf{u}^{h}, \mathbf{v}^{h}\right)+b\left(p^{h}, \mathbf{v}^{h}\right) & =\left(\mathbf{f}, \mathbf{v}^{h}\right)+\int_{\partial \Omega_{N}} \mathbf{g}_{N} \cdot \mathbf{v}^{h} d s & & \forall \mathbf{v}^{h} \in V^{h} \\
b\left(q^{h}, \mathbf{u}^{h}\right)+c_{1}^{h}\left(p^{h}, q^{h}\right) & =c_{2}^{h}\left(\mathbf{u}^{h}, q^{h}\right) & & \forall q^{h} \in Q^{h}
\end{aligned}
$$

where

$$
\begin{aligned}
c_{1}^{h}\left(p^{h}, q^{h}\right) & =\nu^{-1} \sum_{K \in \mathcal{P}} h_{K}^{2}\left(\nabla p^{h}, \nabla q^{h}\right)_{K} \\
c_{2}^{h}\left(\mathbf{u}^{h}, q^{h}\right) & =\nu^{-1} \sum_{K \in \mathcal{P}} h_{K}^{2}\left(\mathbf{f}+\nu \Delta \mathbf{u}^{h}, \nabla q^{h}\right)_{K} .
\end{aligned}
$$

The estimator (5.15) should be modified accordingly, and it can be shown, see [3], that the natural extension is to multiply the first of the three terms in the estimator by a factor of 2. This still gives a robust estimator in the sense of (5.9) and (5.10).

\subsection{Example 1}

The first example is flow between two concentric cylinders where the outer cylinder is kept fixed and the inner cylinder rotates with angular velocity $\omega_{0}=1$. The radii of the cylinders are $r_{0}=4$ and $r_{1}=9$. Here the mixed triangular element is used.

Denoting by $u_{\theta}$ and $u_{r}$ the angular and the radial velocity, the analytical solution is known to be

$$
\begin{aligned}
u_{\theta} & =r_{0} \omega_{0} \frac{r_{1} / r-r / r_{1}}{r_{1} / r_{0}-r_{0} / r_{1}} \\
u_{r} & =0 \\
p & =p_{0}=\text { const }
\end{aligned}
$$

We will compute the torque, around the cylinder centre, on the outer cylinder. This is obtained by choosing the extraction field as

$$
\left.\mathbf{w}_{u}\right|_{r=r_{1}}=-\left(y-y_{c}\right)[1,0]^{T}+\left(x-x_{c}\right)[0,1]^{T} \quad \text { for } \quad x^{2}+y^{2}=r_{1}^{2},
$$



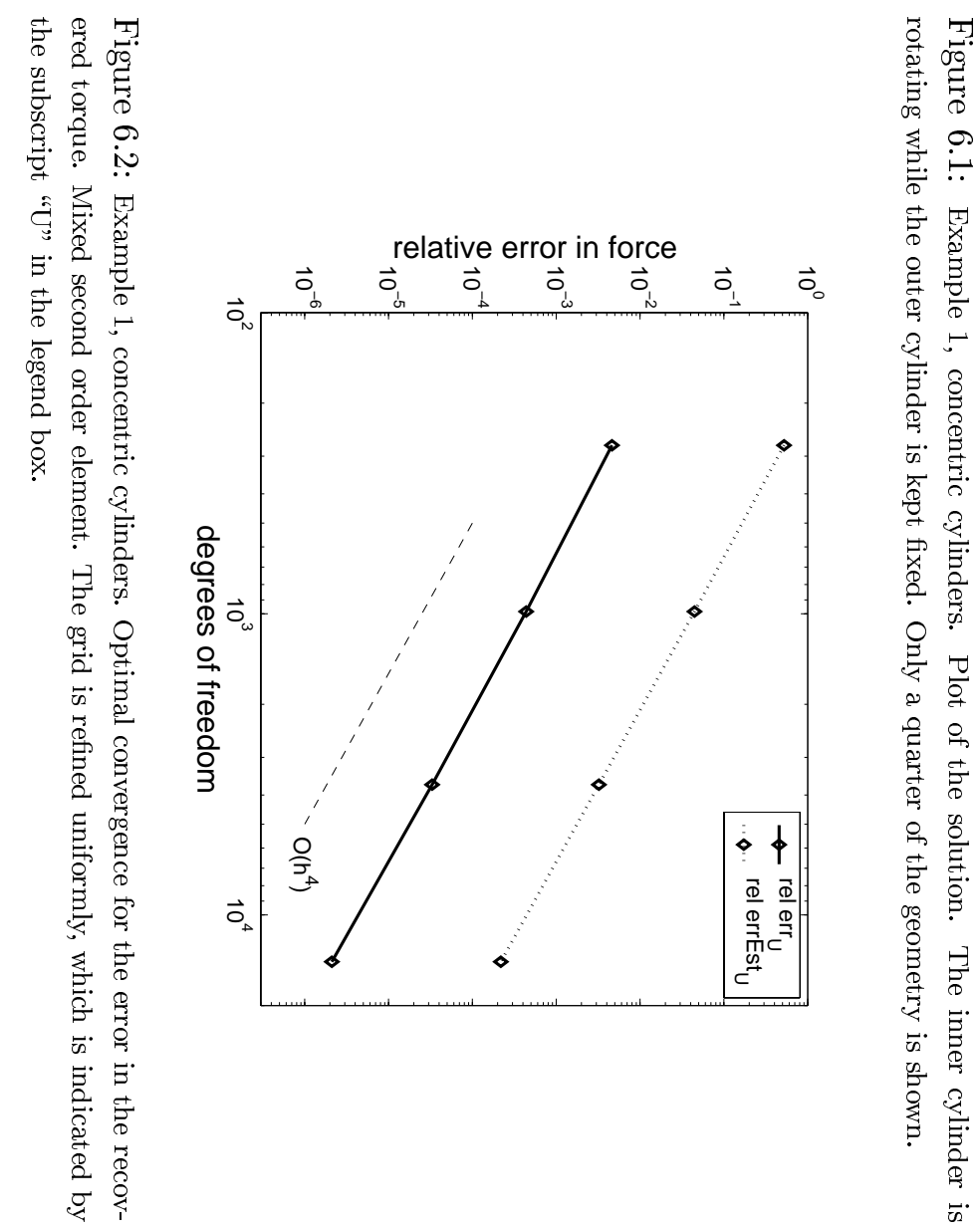

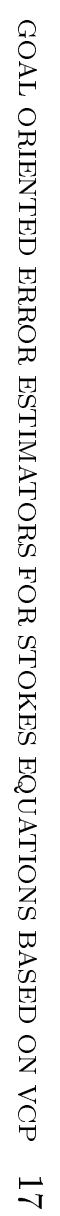




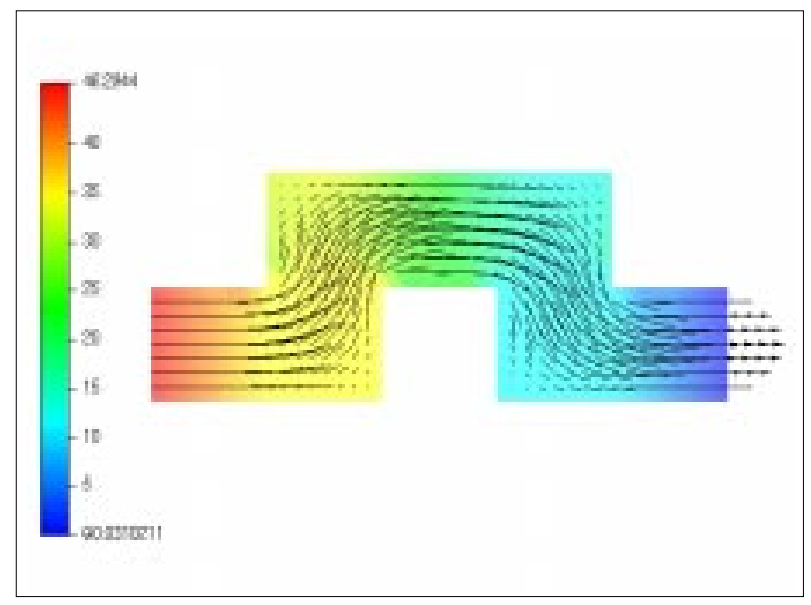

Figure 6.3: Example 2, flow through a bent pipe. Plot of the solution. The maximum velocity at the inlet is 1 and all the short boundary segments have length 1 . The shading shows the pressure distribution.

where $\left(x_{c}, y_{c}\right)$ is the cylinder centre. The above expression is easily found from letting $\mathbf{w}_{u}=\mathbf{t}$ and then multiplying by the distance from the cylinder centre. From the analytical solution the exact value for the torque $T$ is found to be

$$
T=\frac{4 \pi r_{1}^{2} \omega_{0}}{r_{1}^{2} / r_{0}^{2}-1}
$$

This example has a smooth solution, and we use uniformly refined grids to show that the optimal convergence rate is obtained for the actual error associated with the VCP procedure and for the estimated error computed from (5.12) and (5.15). Since second order elements are applied we expect the energy norm of both the primal and the dual solution to converge as $\mathcal{O}\left(h^{2}\right)$, and the error in the torque is therefore expected to converge as $\mathcal{O}\left(h^{4}\right)$. Figure 6.1 shows a quarter of the geometry and the solution. The convergence results are shown in Figure 6.2.

\subsection{Example 2}

In the second example a fluid runs through a bent pipe. All boundary segments are walls except the inlet, where a parabolic inflow profile is given, and the outlet where there is a homogeneous Neumann condition, see Figure 6.3. The force to be recovered is the shear force on the lower wall next to the inlet, and the "exact" value for the force was found from a solution on a very fine grid $\left(3.5 \cdot 10^{5}\right.$ degrees of freedom), giving $F_{e x} \approx 5.42758$. For the recovery of this force an extraction field of the type (4.3) with $\mathbf{d}$ equal to the tangent vector is applied. The tangent has only one component and hence the extraction field may be chosen as in Figure 6.4. The shear force on the wall will be computed using 


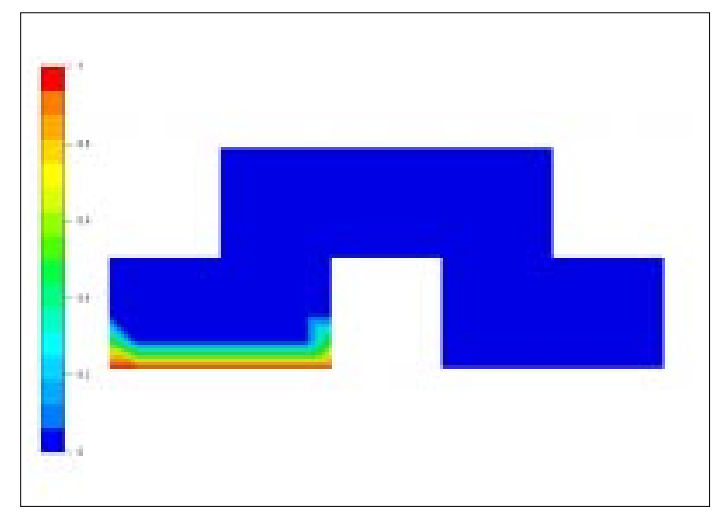

Figure 6.4: The extraction field for computing the shear force acting on the wall. Only the first component is shown since the second component is zero. The field is unity at the wall and it vanishes outside a domain close to the wall. The lack of symmetry is due to grid effects.

the recovery technique described in previous sections and the refinement of the grid will be done in four different ways: Uniform refinement, refinement based on the energy error estimator (5.15), refinement based on the goal oriented error indicator (5.12), and refinement based on the goal oriented error indicator (5.16). Refinements are done by refining the $25 \%$ of the elements that have the largest error indicators. In this example first order stabilised triangular elements are applied.

Figure 6.5 shows the resulting convergence plots. There the relative error in the force is plotted against degrees of freedom, and the different refinement strategies are compared. In the same plot also the corresponding error indicators are plotted as dotted lines. The error indicators plotted are in all cases $\left(\eta^{*}\right)^{2}$ with $\eta_{K}^{*}$ as in (5.16), even if this is usually not computed unless the goal oriented strategy is applied. The only exception is when $\eta_{K}$ defined in (5.12) is applied for adapting the grid. Then the dotted line is $\eta^{2}$. This is shown in the following table.

\begin{tabular}{c|cc}
$\begin{array}{c}\text { Estimator used } \\
\text { for refinement }\end{array}$ & $\begin{array}{c}\text { Subscript } \\
\text { in legend }\end{array}$ & $\begin{array}{c}\text { Estimator } \\
\text { plotted }\end{array}$ \\
\hline uniform & $\mathrm{U}$ & $\left(\eta^{*}\right)^{2}$ \\
$\hat{\eta}_{K}$ & $\mathrm{E}$ & $\left(\eta^{*}\right)^{2}$ \\
$\eta_{K}$ & $\mathrm{GO}$ & $\eta^{2}$ \\
$\eta_{K}^{*}$ & $\mathrm{GO}^{*}$ & $\left(\eta^{*}\right)^{2}$
\end{tabular}

Note that the reason for plotting $\left(\eta^{*}\right)^{2}$ when uniform refinement or refinement according to the energy estimator is applied, is that in these cases the refinement criterion says nothing about the error in the force. Usually the plotted quantity is not available in these cases.

Figure 6.5 shows that all strategies give close to optimal convergence rate for 


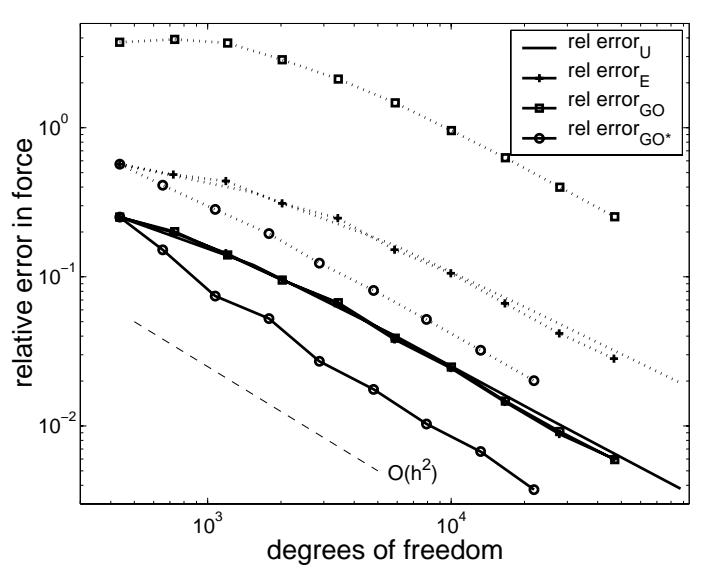

Figure 6.5: Example 2, flow through a bent pipe. Convergence plot of the error in the recovered force for the stabilised first order elements. The different errors are plotted with solid lines and the corresponding estimators are plotted with dotted lines.

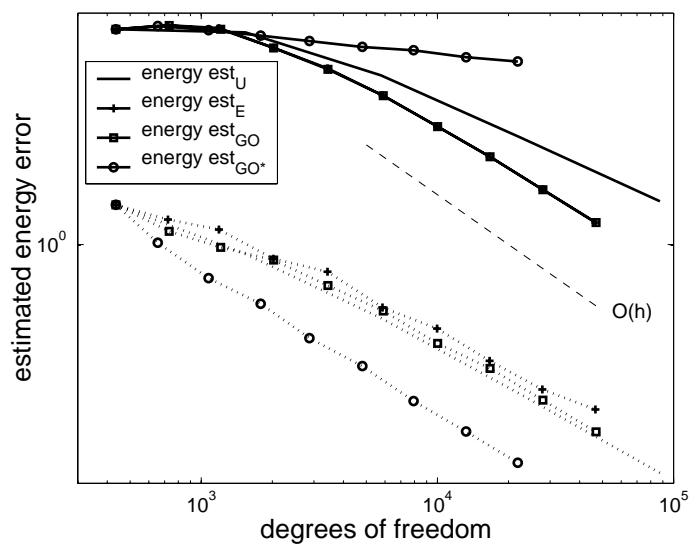

Figure 6.6: Example 2, flow through a bent pipe. Convergence plot of the energy error estimators for the primal and the dual problem when using the different adaptive strategies. The estimators for the primal problem are plotted with solid lines, the estimators for the dual problem are plotted with dotted lines.

the recovered force. The estimator $\eta^{*}$ gives the best result, and to get the relative error down to $10^{-2}$ the other strategies require approximately 3.5 times as many degrees of freedom as the $\eta^{*}$-strategy. Also the estimators clearly show this difference in quality between the $\eta^{*}$-strategy and the other strategies.

Although it over estimates the error by a large factor it is worth noticing that the 
a)

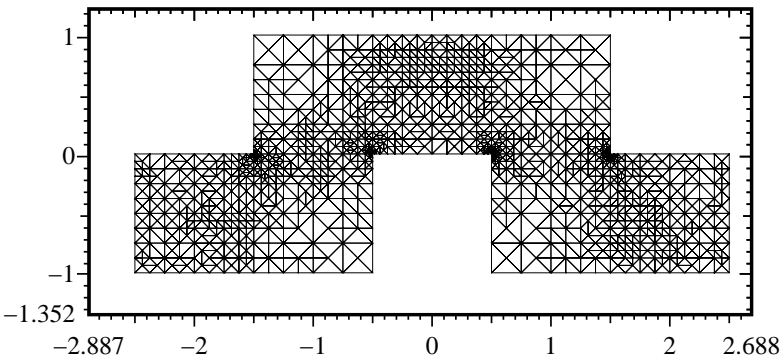

b)

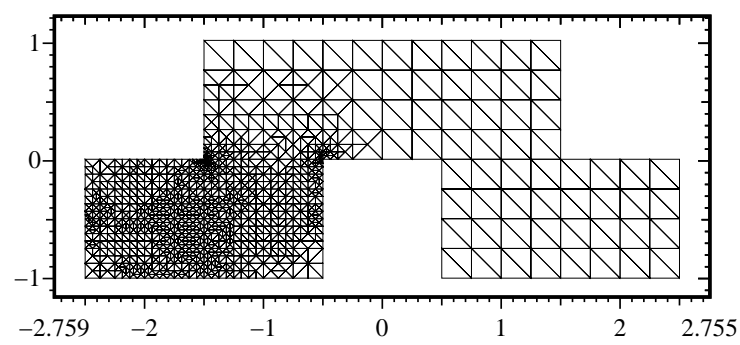

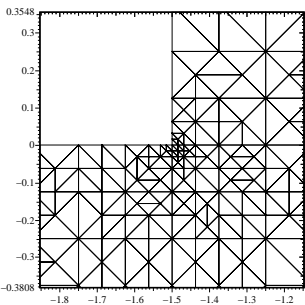

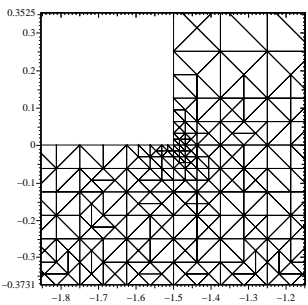

Figure 6.7: Example 2, flow through a bent pipe. Plot a) shows the grid produced after 5 refinements using the energy error estimator, $\hat{\eta}_{K}$. Plot b) shows the grid after 5 refinements using the estimator $\eta_{K}^{*}$. In both cases the picture on the right shows an enlargement of one of the re-entrant corners.

$\eta$-strategy gives an estimator which converges with (almost) optimal rate. This is (almost) guaranteed since the energy error for the primal problem, which has the dominant errors, has (almost) optimal convergence rate for the corresponding grids. This can be seen from Figure 6.6 where the robust energy error estimators are plotted for the primal and the dual problem. Because of the singularities the energy error in the primal problem does not obtain an optimal convergence rate when uniform refinement is applied. However, this does not seem to affect the convergence of the recovered force. Also the estimator $\eta^{*}$ does not obtain optimal convergence in energy norm for the primal problem, which is expected since it disregards large errors in the primal problem in areas where the dual error is small. Examples of the grids produced by $\hat{\eta}_{K}$ and $\eta_{K}^{*}$ can be seen in Figure 6.7.

\subsection{Example 3}

The third example is flow around a cylinder in a channel with no-slip walls. This is a test problem that was suggested for Navier-Stokes solvers in [9], and 


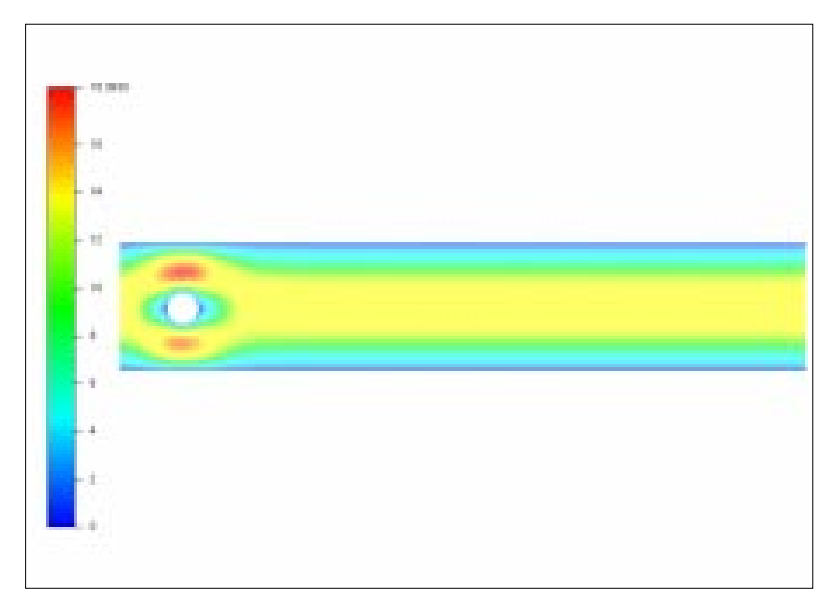

Figure 6.8: Example 3, flow around a cylinder in a channel. The figure shows the geometry and the magnitude of the velocity.

the geometry is taken from there:

$$
\begin{aligned}
\text { cylinder diameter } & =0.1 \\
\text { width of channel } & =0.41 \\
\text { length of channel } & =2.2 \\
\text { cylinder centre } & =(0.2,0.2)
\end{aligned}
$$

The inflow is given as a parabolic profile with maximum velocity $u_{0}=15$. At the outlet there is a homogeneous Neumann condition, and as previously $\nu=1$. The solution is shown in Figure 6.8, where the first order stabilised element has been applied. In the example the drag force acting on the cylinder is computed. The "exact" force was computed on a fine grid, and it was found to be $F_{e x} \approx 314.2$. Refinements are done by refining the $25 \%$ of the elements that have the largest error indicators.

The results are shown in Figure 6.9 and 6.10. The plots show that all estimators and the corresponding errors obtain close to optimal rate of convergence. As in the previous example the error in the primal variables do not obtain optimal rate of convergence when $\eta_{K}^{*}$ is used in the adaptive procedure. Again this is natural, since the dual weights allow a large error in domains that are not important for the accuracy of the recovered force. However, this goal oriented estimator clearly gives the the smallest actual error, and also the smallest error estimate. The grids produced by $\hat{\eta}_{K}$ and $\eta_{K}^{*}$ are found in Figure 6.11 . 

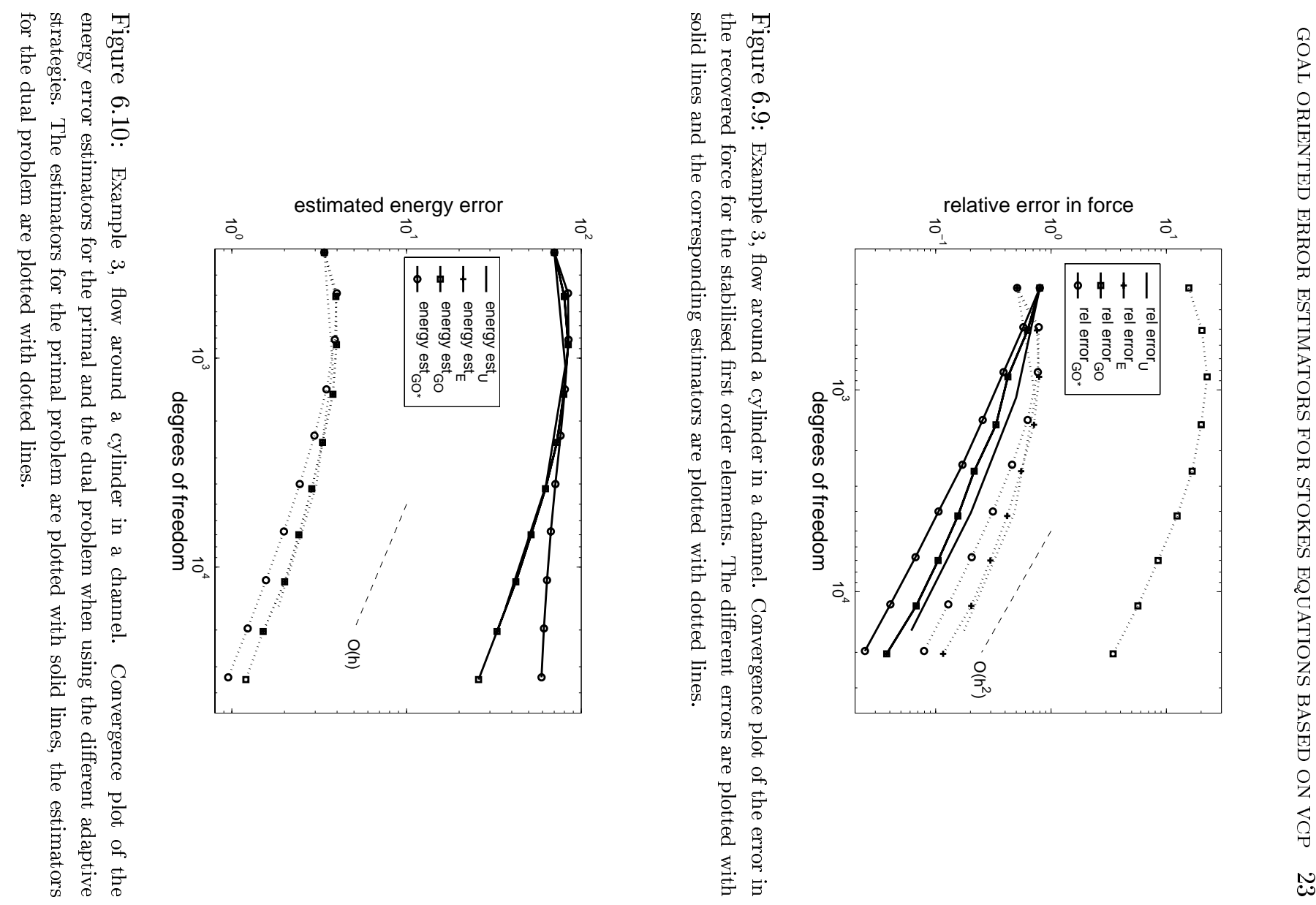
a)
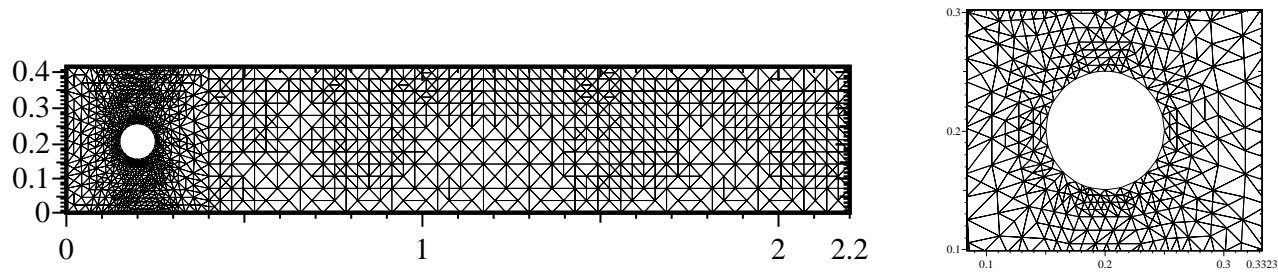

b)
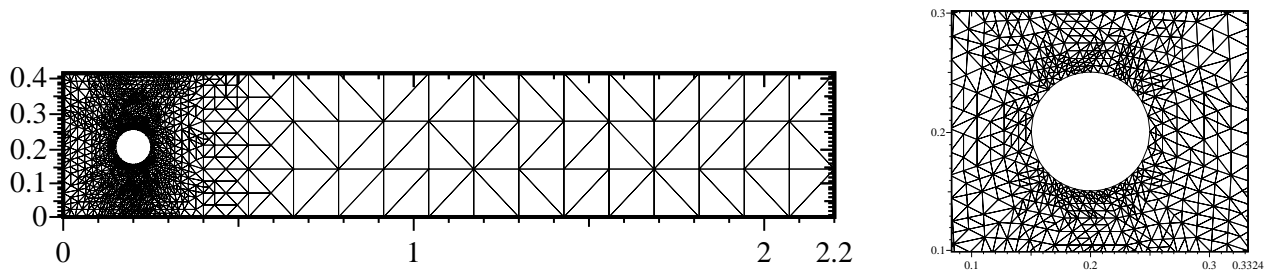

Figure 6.11: Example 3, flow around a cylinder in a channel. Plot a) shows the grid produced after 5 refinements using the energy error estimator, $\hat{\eta}_{K}$. Plot b) shows the grid after 5 refinements using the estimator $\eta_{K}^{*}$. In both cases the picture on the right shows an enlargement of the grid close to the cylinder.

\subsection{Example 4}

The final example is identical to the second example, but this time the mixed triangular element is applied. For this element no stabilisation is needed. Again refinements are done by refining the $25 \%$ of the elements that have the largest error indicators.

The results are shown in Figure 6.12. First note that uniform refinement gives a lower rate of convergence than refining the grid according to either of the three adaptive strategies. In this example the plots clearly show that uniform refinement is not an optimal strategy. All the adaptive strategies obtain almost the same rate of convergence. However, when $\eta_{K}^{*}$ is applied one needs less than $1 / 4$ of the degrees of freedom used by the other adaptive strategies to get the relative error down to a certain level. The estimator also indicates the smallest error when the $\eta^{*}$-strategy is applied. All the adaptive strategies obtain close to optimal convergence rate, and the estimators converge with the same rate as the actual errors. 


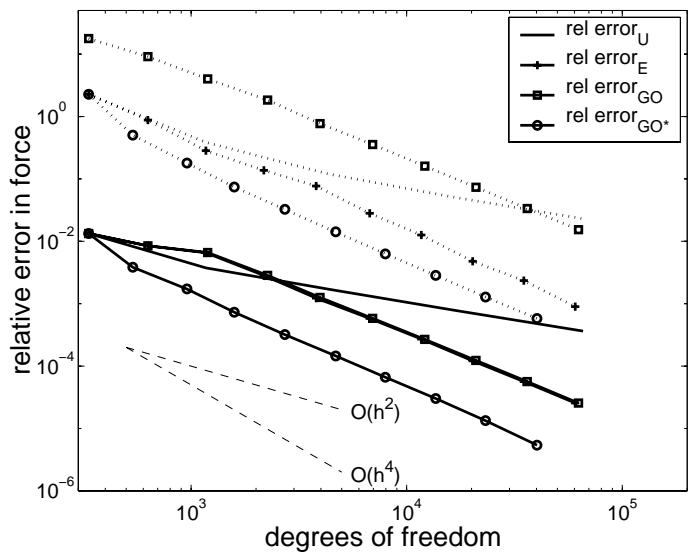

Figure 6.12: Example 4, flow through a bent pipe. Convergence plot of the error in the recovered force for the second order elements. The different errors are plotted with solid lines and the corresponding estimators are plotted with dotted lines.

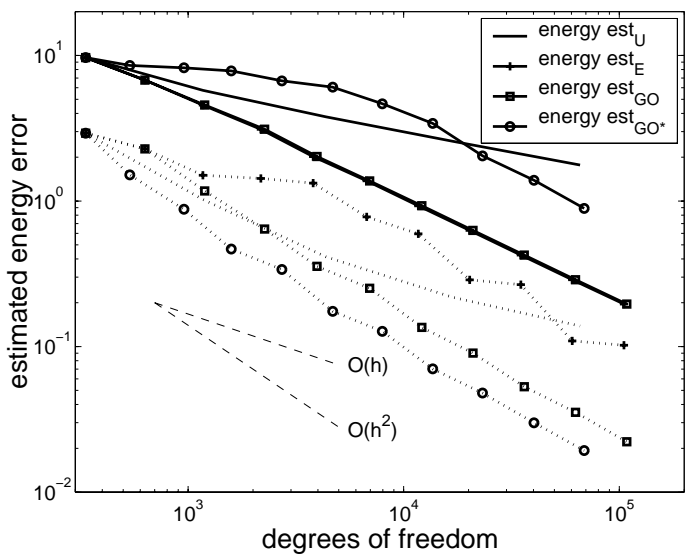

Figure 6.13: Example 4, flow through a bent pipe. Convergence plot of the energy error estimators for the primal and the dual problem when using the different adaptive strategies. The estimators for the primal problem are plotted with solid lines, the estimators for the dual problem are plotted with dotted lines.

Figure 6.13 shows a plot of the energy estimator, $\hat{\eta}$, evaluated for the primal and the dual problem. It is clear that uniform refinement does not give an optimal rate of convergence. Also, similar to the plots for the previous examples, $\eta^{*}$ seems to keep the error in the dual problem at a lower level than what is obtained by the other strategies. The grids produced by $\hat{\eta}_{K}$ and $\eta_{K}^{*}$ are very similar to those shown in Figure 6.7. 


\section{References}

[1] M.Ainsworth and J.T.Oden. A posteriori error estimation in finite element analysis. Computational methods in applied mechanics and engineering 142, 1-88. Elsevier Science S.A., 1997.

[2] I.Babuška and A.Miller. The post-processing approach in the finite element method - Part 1: Calculation of displacements, stresses and other higher derivatives of the displacements. International Journal for Numerical Methods in Engineering, 20, 1085-1109, 1984.

[3] R.Becker. Weighted Error Estimators for Finite Element Approximations of the Incompressible Navier-Stokes Equations. Preprint 98-20, SFB 359, Universität Heidelberg, 1998.

[4] R.Becker and R.Rannacher. A feed-back approach to error control in finite element methods: Basic analysis and examples. East-West J.Numer. Math., 4, pp. 237-264, 1996.

[5] S.C.Brenner and L.R.Scott. The Mathematical Theory of Finite Element Methods. Springer-Verlag, New York, 1994.

[6] M.Giles, M.G.Larson, J.M.Levenstam, and E.Süli. Adaptive error control for finite element approximations of the lift and drag coefficients in viscous flow. Tech. report NA-97/06, Oxford University Computing Laboratory, Oxford, UK, 1997.

[7] T.Kvamsdal. Variationally Consistent Postprocessing. In Proceedings for Computational Mechanics, New trends and applications, ed. S.Idelsohn, E.Oñate, and E.Dvorkin, CIMNE, Barcelona, Spain, 1998.

[8] T.Kvamsdal and K.M.Okstad. Error Estimator for Variational Consistent Surface Forces in Navier-Stokes Simulations. In Proceedings for Computational Mechanics, New trends and applications, ed. S.Idelsohn, E.Oñate, and E.Dvorkin, CIMNE, Barcelona, Spain, 1998.

[9] S.Turek. A comparative study of some time-stepping techniques for the incompressible Navier-Stokes equations: From fully implicit nonlinear schemes to semi-implicit projection methods. Int. J. Numer. Meth. Fluids, 22, pp. 987-1011, 1996.

[10] R.Verfürth. A review of a posteriori error estimation and adaptive meshrefinement techniques. Wiley and Teubner, Germany, 1996. 